\title{
The effects of two different intensities of aerobic training protocols on pain and serum neuro-biomarkers in women migraineurs: a randomized controlled trail
}

\author{
Eslami, Rasoul ; Parnow, Abdolhossein ; Pairo, Zahra ; Nikolaidis, Pantelis ; Knechtle, Beat
}

\begin{abstract}
Objectives: We have a weak understanding of how aerobic training may influence migraine, and the optimal parameters for exercise regimens as migraine therapy are not clear. The objectives of this study were to assess, first, effects of two different intensities of aerobic exercise on migraine headache indices; second, serum neuro-biomarker in women migraineurs. Methods: A total of 45 non-athlete female migraine patients were selected by a neurologist and randomly divided into three groups: control (CON), moderate-intensity aerobic training (MOD T), and high-intensity aerobic training (HIGH T). Before and after the training protocol, body composition factors, migraine pain indices, VO2max, and serum Adenylate-Cyclase Activating Polypeptide (PACAP) and Substance P (SP) were measured. Exercise training protocol includes two different intensities of aerobic exercise: Moderate (13-15 Borg Scale, 60-80\% HRmax) and High (15-17 Borg Scale, 65-95\% HRmax). Results: Moderate-intensity aerobic training (MOD T) reduced headache intensity, frequency, and duration in women with migraine $(\mathrm{p}<0.001$, for all). Also, high-intensity aerobic training (HIGH T) reduced headache intensity, frequency, and duration ( $\mathrm{p}<0.001$, for all). However, for headache intensity and duration, MOD T was effective rather than HIGH T ( $\mathrm{p}<0.001 ; \mathrm{p} \leq 0.05$, respectively). In addition, neither MOD T nor HIGH T could not alter PACAP and SP contents ( $\mathrm{p}=0.712 ; \mathrm{p}=0.249$, respectively). Conclusions: Our results demonstrated that either MOD T or HIGH T could modify migraine pain indices but neither MOD T nor HIGH T could not alter the $\mathrm{PACAP}$ and SP contents in women with migraine.
\end{abstract}

DOI: https://doi.org/10.1007/s00421-020-04551-x

Posted at the Zurich Open Repository and Archive, University of Zurich

ZORA URL: https://doi.org/10.5167/uzh-192070

Journal Article

Accepted Version

Originally published at:

Eslami, Rasoul; Parnow, Abdolhossein; Pairo, Zahra; Nikolaidis, Pantelis; Knechtle, Beat (2021). The effects of two different intensities of aerobic training protocols on pain and serum neuro-biomarkers in women migraineurs: a randomized controlled trail. European Journal of Applied Physiology, 121(2):609-620.

DOI: https://doi.org/10.1007/s00421-020-04551-x 


\section{The Effects of Two Different Intensities of Aerobic Training \\ 2 Protocols on Pain and Serum Neuro-biomarkers in Women \\ 3 Migraineurs: A Randomized Controlled Trail}

4

Different intensities of aerobic training and migraine pain

Rasoul Eslami ${ }^{1, \mathrm{a}}$, Abdolhoseein Parnow ${ }^{2, \mathrm{a}}$, Zahra Pairo ${ }^{2}$, Pantelis Nikolaidis ${ }^{3}$, Beat Knechtle ${ }^{4}$ 5

${ }^{1}$ Faculty of Sport Science, AllamehTabataba'i University, Tehran, Iran

${ }^{2}$ Exercise Physiology Department, Faculty of Sport Sciences, Razi University, Kermanshah, Iran

${ }^{3}$ Exercise Physiology Laboratory, Nikaia, Greece

${ }^{4}$ Institute of Primary Care, University of Zurich, Zurich, Switzerland

${ }^{5}$ Medbase St. Gallen Am Vadianplatz, St. Gallen, Switzerland

${ }^{\mathrm{a}} \mathrm{RE}$ and AP contributed equally.

Rasoul Eslami

Abdolhoseein Parnow

Zahra Pairo

Pantelis Nikolaidis

Beat Knechtle eslami.rasul@gmail.com

parnowabdolhossein@gmail.com

zahrapairo@gmail.com

pademil@hotmail.com

beat.knechtle@hispeed.ch

\section{Corresponding author}

Prof. Dr. med. Beat Knechtle

Medbase St. Gallen Am Vadianplatz

Vadianstrasse 26

9001 St. Gallen

Switzerland

Telephone +41(0) 712269300

Telefax +41(0) 712269301

E-mail beat.knechtle@hispeed.ch 


\section{Abstract}

43 Objectives: We have a weak understanding of how aerobic training may influence migraine, and

44 the optimal parameters for exercise regimens as migraine therapy are not clear. The objectives of

45 this study were to assess, first, effects of two different intensities of aerobic exercise on migraine

46 headache indices; second, serum neuro-biomarker in women migraineurs.

47 Methods: A total of 45 non-athlete female migraine patients were selected by a neurologist and 48 randomly divided into three groups: control $(\mathrm{CON})$, moderate-intensity aerobic training (MOD

$49 \mathrm{~T}$ ), and high-intensity aerobic training (HIGH T). Before and after the training protocol, body 50 composition factors, migraine pain indices, $\mathrm{VO}_{2} \max$, and serum Adenylate-Cyclase Activating

51 Polypeptide (PACAP) and Substance P (SP) were measured. Exercise training protocol includes

52 two different intensities of aerobic exercise: Moderate (13-15 Borg Scale, 60\% to 80\% HRmax)

53 and High (15-17 Borg Scale, 65\% to 95\% HRmax).

54 Results: Moderate-intensity aerobic training (MOD T) reduced headache intensity, frequency,

55 and duration in women with migraine ( $<<0.001$, for all). Also, high-intensity aerobic training

56 (HIGH T) reduced headache intensity, frequency, and duration ( $p<0.001$, for all). However, for

57 headache intensity and duration, MOD T was effective rather than HIGH T $(\mathrm{p}<0.001 ; \mathrm{p} \leq 0.05$,

58 respectively). In addition, neither MOD T nor HIGH T could not alter PACAP and SP contents

$59(p=0.712 ; p=0.249$, respectively $)$.

60 Conclusions: Our results demonstrated that either MOD T or HIGH T could modify migraine

61 pain indices but neither MOD T nor HIGH T could not alter the PACAP and SP contents in

62 women with migraine. 
63 Key words: Migraine, Aerobic Training, Adenylate-Cyclase Activating Polypeptide (PACAP),

64 Substance P (SP)

65

66

67

68

69

70

71

72

73

74

75

76

77

78

79

80

81

82

83

84

85 


\section{Abbreviations:}

87 BMI: Body Mass Index

88 CGRP: Calcitonin Gene-related Peptide

89 CON: Control

90 CRP: C-Reactive Protein

91 eCB: endocannabinoid

92 HRmax: Heart Rate Maximum

93 HIGH T: High-intensity aerobic training

94 IL-6: Interleukine-6

95 NPY: Neuropeptide Y

96 NO: Nitric Oxide

97 MOD T: Moderate-intensity aerobic training

98 PACAP: Pituitary Adenylate-Cyclase Activating Polypeptide

99 RCT: Randomized Controlled Trial

100 SNL: Spinal Nerve Ligation

101 SP: Substance P

102 VAS: Visual Analog Scale

103 Vo2max: Maximum Volume of Oxygen Consumption

104 WHR: Waist-to-Hip Ratio

105

106

107

108 


\section{Introduction}

111 Migraine is a debilitating neurovascular disorder characterized by severe headache attacks often

112 associated with autonomic and neurological symptoms (Ferrari, 1998; Goadsby, Lipton, \&

113 Ferrari, 2002). Depression, chronic fatigue, disability on work, family problems, and drug

114 dependency are some psychosocial problems related to these chronic headaches (Gantenbein,

115 Afra, Jenni, \& Sándor, 2012). It is well established that migraine headache has a higher

116 prevalence in females than males. Many studies with a variety of samples and methodologies

117 revealed that females have a higher prevalence ratio in migraines than males (Benbir et al, 2012;

118 Henry et al,1999; Kim et al, 2013; Buse et al, 2013). Also, females may report more symptoms

119 and headache-related disability (Benbir et al, 2012; Henry et al,1999; Steiner et al, 2003).

120

121 The lack of suitable therapeutic modalities in managing migraine reflects the limited

122 understanding of the pathological mechanisms behind it. According to the neurovascular theory

123 of migraine, several neuropeptides may play a role in trigemino-neurovascular system activation,

124 which has been considered as one of the important migraine pathomechanism (Tajti, Szok,

125 Majláth, Tuka, \& Csáti, 2015). Several studies in this regard have reported the role of

126 neuropeptides, including calcitonin gene-related peptide (CGRP), Nitric Oxide (NO),

127 Neuropeptide Y (NPY), Pituitary Adenylate-Cyclase Activating Polypeptide (PACAP) and

128 Substance P (SP), in migraine pathophysiology (Tajti et al., 2015).

129

130 Adenylate-Cyclase Activating Polypeptide (PACAP) is a multifunctional vasodilator peptide that

131 has recently been shown to play an important role in migraine pathogenesis, such as CGRP. A 
132 new clinical study has provided evidence of a clear association between migraine phases (ictal

133 and interictal) and plasma PACAP alterations in migraine (Tuka et al., 2013). This study showed

134 that the plasma levels of PACAP were increased during migraine attacks compared to their

135 interictal levels, while interictal PACAP levels were lower in comparison to healthy subjects

136 (Tuka et al., 2013). Also, peripheral injection of PACAP in migraineurs, induced a delayed

137 migraine-like headache, while PACAP injection in healthy subjects led to initial headache

138 (Schytz et al., 2009). Therefore, PACAP appears to have the ability to induce a delayed

139 migraine-like headache, such as CGRP.

140

141 SP is a member of the tachykinin-neuropeptide family (Chang, Leeman, \& Niall, 1971). During

142 activation of the trigemino-neurovascular system, SP induces vasodilatation and plasma protein

143 extra vacation in the cerebral dura mater (Buzzi \& Moskowitz, 1990; Moskowitz, 1993). In the

144 clinical situation, the salivary SP immunoreactivity was elevated during spontaneous migraine 145 attacks without aura when compared with the control subjects (Nicolodi \& Del Bianco, 1990).

146 Also, in chronic migraine patients, the saliva and plasma levels of SP were higher than those in

147 healthy subjects, and associated with pain intensity (Jang, Park, Kho, Chung, \& Chung, 2011).

148 Therefore, recent evidence focuses on the possible role of PACAP and SP in migraine

149 pathophysiology.

151 Aerobic (endurance) exercise is considered as a therapeutic method of reducing symptoms of 152 several chronic diseases and medical conditions (Scheef et al., 2012). As direct evidence, the 153 therapeutic effects of endurance training on migraine have been reported in numerous studies. A 154 large body of literature addressing exercise may be particularly helpful in reducing the 
155 frequency, duration, and intensity of migraine headaches (Darabaneanu et al., 2011; Varkey E,

156

157

158

159

160

161

162

163

164

165

166

167

168

169

170

171 training in the migraine society and the unknown mechanism involved in this process are strong

172 reasons why future investigations should be performed. Consequently, the purpose of the current

173 study was to estimate the effects of two different aerobic protocols on headache indices and to

174 investigate the behavior of serum levels of biological markers (PACAP and SP) playing a key

175 role in pain mechanisms, after aerobic training protocols in women migraineurs. The research

176 hypothesis, therefore, was that the intensity of training is a main variable to determine a positive 
177 effect of MOD and HIGH aerobic training on headache indices and to be effective to decrease 178 migraine pain indexes in migraine patients.

179

180

181

182

183

184

185

186

187

188

189

190

191

192

193

194

195

196 


\section{Material and Methods}

\section{Research design}

200 This was a randomized controlled clinical trial (RCT) with a semi-experimental design (three 201 groups in the form of pre-test and post-test) (RCT code: IRCT2015100910824N2) and was

202 conducted among the migraine patient's community in Kermanshah city, Iran. The experimental 203 procedures and study protocols were approved by the Ethics Committee of Kermanshah 204 University of Medical Sciences (ethical code: kums.rec.1394.15).

In order to determine the number of subjects, the randomized sampling method was used.

207 Volunteers were recruited from neurology clinics, and neurology center of Kermanshah hospital 208 (Kermanshah, Iran). The inclusion criteria were as follows: (1) Not having a history of regular 209 exercise in the last six months, (2) having no cardiovascular disease, hypertension, diabetes, 210 arthritis, cancer, asthma, AIDS, meningitis and Multiple Sclerosis, (3) confirming headache by 211 the neurologist, and (4) obtaining the entry criteria for the $\mathrm{VO}_{2} \mathrm{max}$ test (above $20 \mathrm{ml} / \mathrm{kg} / \mathrm{min}$ ).

212 Exclusion criteria were including: (1) situations that researchers have understood during research

213 period such as pregnancy, breastfeeding, abuse of drugs or alcohol; (2) involving in any extra

214 exercise training programs; (3) not interested to continue or change in personal life schedule.

215 According to the study criteria for subject selection, 45 non-athlete female migraine patients 216 were selected by the neurologist. Prior to participation, written consent was obtained from all 217 patients. The subjects who met the inclusion criteria were randomly assigned into three groups: 218 control $(\mathrm{CON})(n=15)$, moderate-intensity aerobic training (MOD T) $(n=15)$, and high-intensity 219 aerobic training (HIGH T) $(n=15)$ (Figure 1). The initial demographic characteristics of the 
220 subjects for the different groups are shown in Table 1. Patients in Control group (CON) were

221 instructed to keep a diary regarding exercise during the experiments period. Experimental groups

222 included MOD T and HIGH T which MOD T group executed moderate aerobic training and

223 HIGH T group executed high-intensity aerobic training. All three groups received their drug

224 treatment under neurologist supervision.

225 The main protocol of this study lasted for four months: 1 month of migraine evaluation, 2

226 months of training, and 1 month of post evaluation. One month before and after the protocol, the

227 migraine headache indexes (i.e. frequency, duration, and intensity of the attacks) were evaluated

228 by daily headache record questionnaire (Darabaneanu et al., 2011; Narin, Pinar, Erbas, Oztürk, \&

229 Idiman, 2003 ). In addition, $24 \mathrm{~h}$ before and $48 \mathrm{~h}$ after the training protocol, body composition

230 factors (height, age, weight, fat percentage) (In body; Model: Zuse 9.9, South Korea), body mass

231 index (BMI), waist-to-hip ratio (WHR) and $\mathrm{VO}_{2} \max$ (Astrand Bicycle, Monark E839, Sweden)

232 were measured.

\section{Pain evaluation questionnaire}

234 The pain was evaluated using a visual analog scale (VAS). In this scale, the amount of pain that a

235 patient feels ranges across a continuum from none to an extreme amount of pain (the number 0

236 without pain and the number 10 indicates exacerbation of the pain). The mean duration of

237 headache (min per month), as well as the frequency of headache (days per month) was evaluated

238 using this questionnaire (Narin, Pinar, Erbas, Oztürk, \& Idiman, 2003 ).

239

240

241 
243 One day before starting training protocols and $48 \mathrm{~h}$ after the last training sessions, blood samples

244 were collected from an cubital vein at the same time in the morning and after a $12 \mathrm{~h}$ fasting state.

245 For all groups, blood samples were collected outside a migraine attack and having taken no

246 symptomatic medication the day before. Blood samples were immediately centrifuged at a

247 temperature of $4{ }^{\circ} \mathrm{C}$ and $3000 \mathrm{rpm}$ for $10 \mathrm{~min}$. Thereafter, plasma was separated and kept at -80

$248{ }^{\circ} \mathrm{C}$ until analysis. Plasma levels of PACAP and SP were measured by commercially available

249 enzyme-linked immunosorbent assay kits (Kazabu Co, Japan, with catalog number CSB-

250 E09348h for PACAP; R \& D Co, Canada, with catalog number KGE007 for SP) according to the

251 procedures provided by the manufacturer.

252

253

Exercise Training protocols

254 As mentioned above, in this study, we have two experimental groups. These two groups not only 255 received their drug treatment but were also treated with aerobic training including MOD T and 256 HIGH T. As shown in Table 2, the MOD T protocol (13-15 Borg Pressure Understanding Scale, $25760-80 \%$ heart rate max, equivalent to 45\%-70 \% VO 2 max) and HIGH T protocol (15-17 Borg 258 Pressure Understanding Scale, 65-95 \% heart rate max, equivalent to 55\% - 90\% VO 2 max) were 259 designed from a simple to heavy state. A simple or heavy state was declared by subjects 260 according to training pressure. The training programs included eight weeks, three sessions per 261 week, 30-60 min per session including warm up and cool down. For both training groups, every 262 training session was divided into three parts: A. warming up (10 min), B. main training 263 (according to weekly duration), and C. cooling down (10 min) (Table 2). 
266 It is important to mention that during study, all patients used and monitored migraine

267 medications according to expert neurologist. Based on initial assessment by expert neurologist,

268 needed dose of drug (including Diclofenac, Nortriptyline and Duloxetine, Sumatriptan, Depakin,

269 Propranolol, Dexamethazone, Venlafaxine, Gabapentin, Ergotamine, Zolpidem, Codein,

270 Ibuprofen) was administered to all patients and dose of drug used were recorded during study.

271

272 Statistical analysis

273 Data were expressed as mean \pm SD. Distribution of data was assessed for normality using the

274 Shapiro-Wilk test. The data were normally distributed, therefore, were analyzed using

275 parametric methods. A 2 (Time) x 3 (Group) mixed-model of Repeated measures ANOVA test,

276 followed by paired sample t-test (for pre-post comparison in every group) and Scheffe post hoc

277 test (for between-groups comparison) were used. The differences were accepted as significant if

$278 \mathrm{p} \leq 0.05, \mathrm{p} \leq 0.01$, or $\mathrm{p} \leq 0.001$.

279

280

281

282

283

284

285

286 


\section{Results}

A 2 (Time) x 3 (Group) mixed-model ANOVA for weight data analysis revealed that the main

291 effect for time was not significant $(\mathrm{F}(1,30)=4.934, \mathrm{p}=0.081$, Eta-squared $=0.098)$. This means

292 weight was not different between pre and post time, for three groups. Also, no significant main

293 effect for group was obtained $(\mathrm{F}(1,30)=2.851, \mathrm{p}=0.074$, Eta-squared $=0.160)$. Thus, there was

294 no overall difference in the weight between the three groups. However, Time $\mathrm{x}$ group interaction

295 effects was significant $(\mathrm{F}(2,30)=10.157, \mathrm{p}<0.0001$, Eta-squared $=0.404)$. A comparison of

296 means indicated that weight was decreased after aerobic training only in the MOD T group, in

297 comparison to other groups (Table 3).

298 In addition, data analysis of BMI showed that the main effect for group was significant (F $(2,30)$

$299=4.802, \mathrm{p}=0.016$, Eta-squared $=0.243$ ). Thus, there was a significant difference between the

300 three groups. The pairwise comparison test showed that BMI in the MOD T group was

301 significantly higher than the HIGH T group ( $\mathrm{p}<0.05)$. Also, no significant main effect for Time

302 was obtained $(\mathrm{F}(1,30)=3.317, \mathrm{p}=0.079$, Eta-squared $=0.100)$. This means BMI was not

303 different between pre and post time for the three groups. However, Time $\mathrm{x}$ group interaction

304 effects was significant $(\mathrm{F}(2,30)=10.04, \mathrm{p}<0.0001$, Eta-squared $=0.401)$. A comparison of

305 means indicated that BMI decreased after aerobic training only in the MOD T group, in

306 comparison to other groups (Table 3). The results of repeated measures ANOVA for body fat

307 percent revealed a significant main effect for Time $(\mathrm{F}(1,30)=13.59, \mathrm{p} \leq 0.001$, Eta-Squared $=$

308 0.312). Pairwise comparison test showed that in MOD T and HIGH T groups post-test body fat 
309 percent was significantly lower than pre-test ( $<<0.001 ; \mathrm{p} \leq 0.05$, respectively). This indicating that both MOD T and HIGH T were effective in body fat percent reduction in women with

311 migraine disorder (Table 3$)$. In addition, the main effect for group was not significant $(\mathrm{F}(2,30)=$

$3123.290, \mathrm{p}=0.051$, Eta-squared $=0.180)$. Thus, there was no overall difference between the three

313 groups for body fat percent. However, a significant Time x Group interaction effects was also

314 obtained, $\mathrm{F}(2,30)=6.622, \mathrm{p}<0.01$, Eta-squared $=0.306)$. A comparison of means indicated

315 that body fat percent was decreased after aerobic training only in the MOD T group, in

316 comparison to other groups (Table 3).

317 For $\mathrm{VO}_{2}$ max, firstly, it was compared initial $\mathrm{VO}_{2}$ max of patient between three groups via

318 statistical ANOVA test where there was not any significant difference between the three groups

$319(\mathrm{p}=0.088)$. Results showed that there was a significant main effect for Time $(\mathrm{F}(1,30)=35.114$,

$320 \mathrm{p}<0.0001$, Eta-Squared=0.539). The pairwise comparison test showed a significant difference

321 between pre-test and post-test in MOD T and HIGH T groups $(\mathrm{p}<0.001 ; \mathrm{p} \leq 0.001$,

322 respectively). This indicating that both MOD T and $\mathrm{HIGH} \mathrm{T}$ were effective in $\mathrm{VO}_{2}$ max

323 improvement in women with migraine disorder; although the main effect for group was not

324 significant $(\mathrm{F}(2,30)=2.658, \mathrm{p}=0.087$, Eta-squared $=0.151)$. Thus, there was no overall

325 difference between the three groups for $\mathrm{VO}_{2} \max$. However, a significant Time $\mathrm{x}$ Group

326 interaction effects was also obtained, $\mathrm{F}(2,30)=17.434, \mathrm{p}<0.0001$, Eta-squared $=0.538)$. A

327 comparison of means indicated that both MOD T and HIGH T groups had a significant increase

328 in $\mathrm{VO}_{2} \max$ after aerobic training rather, in comparison to the CON group, however, there was

329 not a significant difference between MOD T and HIGH T groups (Table 3). 
330 In addition, for WHR, the main effect for group was not significant $(\mathrm{F}(2,30)=0.628, \mathrm{p}=0.540$,

331 Eta-squared $=0.040)$. Thus, there was no overall difference between the three groups. Also, no

332 significant main effect for Time was obtained $(\mathrm{F}(1,30)=0.657, \mathrm{p}=0.424$, Eta-squared $=0.021)$.

333 This means WHR was not different between pre and post time, for three groups. Moreover, Time

$334 \mathrm{x}$ group interaction effects was not significant $(\mathrm{F}(2,30)=1.626, \mathrm{p}=0.214$, Eta-squared $=0.098)$.

335 Therefore, WHR after the two aerobic training was not different than before aerobic training 336 (Table 3).

\section{$337 \quad$ Migraine pain indexes}

338 A 2 (Time) x 3 (Group) mixed-model ANOVA revealed that there was a strong main effect for

339 Time $(\mathrm{F}(1,29)=62.83, \mathrm{p}<0.001$, Eta-Squared $=0.684)$. pairwise comparison test showed that

340 in MOD T and HIGH T groups, intensity score was significantly lower in post-test rather than

341 pre-test $(\mathrm{p}<0.001 ; \mathrm{p} \leq 0.001$, respectively). This indicated that both MOD T and HIGH T were

342 effective in pain intensity reduction in women with migraine disorder. In addition, the main

343 effect for group was not significant $(\mathrm{F}(2,29)=0.389, \mathrm{p}=0.681$, Eta-squared $=0.026)$. Thus,

344 there was no overall difference between the three groups $(p=0.681)$. However, a significant

345 Time x Group interaction effects was also obtained, F (2,29) =12.241, p < 0.001, Eta-squared =

346 0.458). Comparison of means indicated that, in comparison to the CON group, both MOD T and

347 HIGH T groups had lower intensity the score after aerobic training rather than before aerobic

348 training, however intensity decrease in the MOD T group was larger than HIGH T group. This

349 means moderate-intensity aerobic training was more effective to decrease pain intensity (Table

$3503)$. 
351 In addition, for frequency, there was a strong main effect for Time $(\mathrm{F}(1,29)=85.01, \mathrm{p}<0.001$,

352 Eta-Squared $=0.746)$. The pairwise comparison test showed that, for both MOD T and HIGH T

353 groups, the frequency score in the post-test was significantly lower than the pre-test $(\mathrm{p}<0.001 ; \mathrm{p}$

$354 \leq 0.001$, respectively). This indicated that both MOD T and HIGH T were effective to pain

355 frequency reduction in women with migraine. In addition, the main effect for group was

356 significant $(\mathrm{F}(2,29)=4.411, \mathrm{p}<0.05$, Eta-squared $=0.233)$. The pairwise comparison test

357 showed that the frequency score in MOD T and HIGH T groups was significantly lower than the

358 CON group $(\mathrm{p}<0.01 ; \mathrm{p}<0.05$, respectively). Also, there was not any difference between MOD

359 T and HIGH T groups $(\mathrm{p}=0.462)$. Moreover, a significant Time $\mathrm{x}$ Group interaction effects was

360 also obtained, F $(2,29)=12.241, \mathrm{p} \leq 0.001$, Eta-squared $=0.384)$. Comparison of means

361 indicated that, in comparison to the CON group, both MOD T and HIGH T groups had a lower

362 frequency the score after aerobic training rather than before aerobic training, however, there was

363 not a significant difference between MOD T and HIGH T groups (Table 3).

364 In addition, for Duration, a significant strong main effect for Time was obtained $(\mathrm{F}(1,29)=$

$36554.47, \mathrm{p}<0.001$, Eta-Squared $=0.653)$. The pairwise comparison test showed that, for CON,

366 MOD T, and HIGH T groups, the Duration score in the post-test was significantly lower than the

367 pre-test $(\mathrm{p}<0.001$, for all). This means migraine duration was decreased in three groups. In

368 addition, the main effects for Group was significant $(\mathrm{F}(2,29)=4.872, \mathrm{p}<0.05$, Eta-squared $=$

369 0.252). The pairwise comparison test showed that Duration in the HIGH T group was

370 significantly lower than the CON group $(\mathrm{p}<0.05)$. Also, there was not any difference between

371 MOD T and HIGH T groups $(\mathrm{p}=0.99)$. Moreover, a significant Time $\mathrm{x}$ Group interaction effects

372 was also obtained, $\mathrm{F}(2,29)=3.743, \mathrm{p} \leq 0.05$, Eta-squared $=0.205)$. A comparison of means

373 indicated that both MOD T and HIGH T groups had a significant decrease in duration score after 
374 aerobic training rather than before aerobic training, in comparison to the CON group. However,

375 the decrease in the MOD T group was larger than the HIGH T group. This means moderate-

376 intensity aerobic training was more effective to decrease pain duration (Table 3).

\section{PACAP and SP}

378 For PACAP, a 2 (Time) x 3 (Group) mixed-model ANOVA revealed that the main effect for 379 group was not significant $(\mathrm{F}(2,30)=1.95, \mathrm{p}=0.160$, Eta-squared $=0.115)$. Thus, there was no 380 overall difference between the three groups. Also, no significant main effect for time was

381

382

383

384

385

386

387

388

389

390

391

392 obtained $(\mathrm{F}(1,30)=1.472, \mathrm{p}=0.234$, Eta-squared $=0.047)$. This means PACAP levels were not different between pre and post time, for three groups. Moreover, time $\mathrm{x}$ group interaction effects were not significant $(\mathrm{F}(2,30)=0.344, \mathrm{p}=0.712$, Eta-squared $=0.022)$. Therefore, PACAP after the two aerobic training was not different than before aerobic training (Table 3) (Figure 2).

In addition, data analysis of SP showed that the main effect for group was not significant $(\mathrm{F}(2,30)=0.490, \mathrm{p}=0.618$, Eta-squared $=0.032)$. Thus, there was no overall difference in the SP between the three groups. Also, no significant main effect for Time was obtained $(\mathrm{F}(1,30)=1.43, \mathrm{p}=0.241$, Eta-squared $=0.046)$. This means SP levels were not different between pre and post time, for three groups. Moreover, Time x group interaction effects was not significant $(\mathrm{F}(2,30)=1.456, \mathrm{p}=0.249$, Eta-squared $=0.089)$. Therefore, SP after the 9 two aerobic training was not different than before aerobic training, in comparison to the control 92 group (Table 3) (Figure 2). 
393 The research hypothesis, therefore, was that aerobic training with different intensities could

394 determine the role of volume and intensity of exercise training with a positive effect on headache 395 indices as well as serum levels of PACAP and SP in migraine patients.

396

397

398

399

400

401

402

403

404

405

406

407

408

409

410

411

412 


\section{Discussion}

414 The purpose of the current study was the effects of two different intensities (MOD T and HIGH

$415 \mathrm{~T}$ ) of aerobic training protocols on headache indexes and to investigate them on a serum levels of

416 biological markers (PACAP and SP) in women migraineurs. It was found that, after 8 weeks of

417 MOD T, the intensity (from 7.38 to 3.73 vas), the duration (from 8.68 to 3.6 hours per month),

418 and the frequency (from 7.54 to 2.27 times per month) of migraine headaches decreased and

419 after 8 weeks of HIGH T, the intensity (from 5.79 to 4.22 vas), the duration (from 7.29 to 4.04

420 hours per month), and the frequency (from 8.08 to 3.25 times per month) of migraine headaches

421 reduced.

422 So far, several studies have been conducted regarding exercise training and migraine pain which

423 their results support the positive effects of aerobic training on migraine pain indicators (intensity,

424 duration, and frequency). For example, Kroll et al. (2018) revealed that 45 min of aerobic

425 exercise, 3 times per week, for 3 months lead to a reduction of pain intensity, pain duration, and

426 frequency of migraine attacks. Narin et al. (2003) reported that 8 weeks of aerobic training (3

427 sessions per week and one hour per session) reduced the intensity of migraine headaches (from

4288.8 to 0.4 vas), frequency of headaches (from 4.7 to 3.6 times per month), and duration of

429 headaches (from 37.4 to 11.4 h) in migraine patients (Narin, Pinar, Erbas, Oztürk, \& Idiman,

4302003 ). These researchers attributed the improvement in migraine pain indices to the increase of

431 plasma nitric oxide (Narin et al., 2003 ). Also, Darabaneanu et al. (2013) conducted a study that

432 included an aerobic exercise protocol as running for 10 weeks. They reported a significant

433 decline in headache intensity (from 0.7 to 0.4 vas), headache frequency (from 3.8 to 2.3 times 
434 per month), and duration of headache (from 0.9 to $0.4 \mathrm{~h}$ per month), and these can be related to 435 increase in physical fitness of patients (Darabaneanu et al., 2011 ).

436 However, a number of studies could not demonstrate the effects of aerobic training on migraine 437 headache indices. In terms of the intensity of headache, Loket et al. (1992) and Grimm et al 438 (1981) reported that 6 weeks of aerobic training ( 3 sessions per week, 50 to $85 \% \mathrm{VO}_{2} \max$ ) did 439 not have any positive effect on migraine pain intensity (Grimm, Douglas, \& Hanson, 1981;

440 Lockett \& Campbell, 1992 ). In these two studies, the period of training was 6 weeks that may be 441 too short to produce a sufficient physiological adaptation. In terms of headache duration, 442 Dittrich et al. (2008) did not show the effects of aerobic training on headache duration. Their 443 exercise training program contains 6-week aerobic training with 2 sessions per week, which 444 included 45 min of gymnastics with music and 15 min of relaxation (Dittrich et al., 2008 ). They 445 used a training program with two sessions per week that, according to exercise training 446 principles, cannot induce a complete sensitivity to training. In terms of the frequency of 447 headache attacks, it was reported that 12 weeks of aerobic cycling for three days per week and 20 448 min, each session did not show any significant effect on the frequency of headache attacks 449 (Norlander, Cider, Carlsson, \& Linde, 2007). In this study, the time of training per session was 450 very short $(20 \mathrm{~min})$ that may be insufficient to induce training effects. Therefore, according to 451 the training protocols used in previous studies, it seems that low frequency exercise training per 452 week, short duration exercise per session, short term period (less than 6 weeks), or low total 453 work volume per week (time*frequency) are likely important reasons for the ineffectiveness of 454 these training programs. However, in this study, we an used aerobic training program including 3 455 sessions per week for 8 weeks with 30-40 min per session which thought is sufficient to induce 456 physiological and functional improvement in patient with migraine. 
457 In this study, we tried to investigate about this question "which aerobic training intensity is more 458 appropriate for improving migraine pain indexes?" Therefore, we used two different intensity of 459 aerobic training (MOD T (45-70\% VO 2 max) and HIGH T (55-90\% VO 2 max) with different 460 training volume. The results of this study showed that the intensity of aerobic training was not 461 the main factor in migraine pain control. This is reason why both moderate and high intensity 462 were able to improve the parameters of migraine pain, although the moderate-intensity was 463 somewhat more effective. Therefore, according to the results of this study, it seems that one of 464 the main factors for the success of aerobic programs to improve the Migraine pain indices is its 465 frequency and weekly repetitions. In other words, aerobic training with more than 3 days per 466 week and with moderate to high-intensity levels can be useful for improving migraine pain 467 indicators (intensity, duration, and frequency of headache attacks). In this regard, health 468 guidelines also have reported $150 \mathrm{~min}$ of average intensity aerobic training and $75 \mathrm{~min}$ of high469 intensity aerobic exercises per week with a frequency of 3 to 5 sessions as a useful aerobic 470 training program for health and physical fitness (Kaminsky, 2014). Our results also revealed that

471 MOD T was more effective to decrease migraine pain indexes, although we expect high intensity

472 does it. As the intensity was higher in HIGH T and the volume was higher in MOD T, the

473 workload was calculated via time * RPE and results showed both protocols made exertion the 474 same workload. These results indicate that both MOD T and HIGH T could be an important 475 alternative method in monitoring headache attacks and there is no problem when patients use 476 HIGH T and they could save their time since the time of HIGH T is shorter than MOD T. One of 477 the differences between MOD and HIGH T probably depends on muscle fibers recruitments with 478 showing Slow Oxidative (So) muscle fibers more involved in MOD T and Fast Oxidative 479 Glycolytic (FOG) muscles fibers involved in HIGH T. 
In addition, our results showed that both moderate and high-intensity aerobic training improve

$481 \mathrm{VO}_{2} \max$ and decrease body fat percentage in women with migraine. Also, MOD T could modify

482 weight and BMI in women migraineurs. However, WHR was not affected by neither MOD T nor

483 HIGH T. Thus, aerobic training, especially MOD T, could improve both physical fitness and

484 health factors in women with migraines. Exercise-induced aerobic fitness can affect the

485 physiology of migraine in multiple ways. For example, aerobic training manage weight that

486

result in self-esteem and self-confidence in migraine patients. The body fat percentage reduction

487 also leads to decrease inflammatory factors (e.g., IL-6 and CRP) including in migraine

488 pathophysiology (Irby, et al., 2016). Beyond neurophysiologic pathways, improvements in

489 aerobic fitness and migraine likely are mediated by overlapping alter in behavioral and socio-

490 cognitive factors (e.g., self-efficacy beliefs, outcome expectancies) (Irby et al., 2016; Hindiyeh,

491 Krusz \& Cowan, 2013). However, we run correlation test between $\mathrm{VO}_{2}$ max and migraine

492 indexes in post training, but our results showed no significant correlations.

493

494 There are different mechanisms contributing to the reduction of migraine pain indices after

495 exercise training. For example, Endogenous Opioids System, endocannabinoid (eCB), CGRP,

496 and neurotrophic brain-derived factors may play a significant role in the regulation of pain 497 signaling, which can increase their functions following aerobic exercise training and result in 498 pain relief (Hindiyeh, Krusz, \& Cowan, 2013 ). Beta-endorphin is an endogenous opioid with 499 analgesia effects (Guillemin et al., 1977). Its levels have been found to be lower in patients with 500 migraine in comparison to healthy control. Also, it is lower during migraine attacks than in pain-

501 free periods (Anselmi et al., 1980). It seems that beta-endorphin inhibits the release of SP which 502 results in a lower function of pain pathways (Brunton, 2006). However, exercise can increase 
beta-endorphin levels. In this regard, Köseoglu et al. (2003) studied 6 weeks of aerobic exercise

(40-50 min 3 times per week at $60-80 \%$ of maximal heart rate) on female migraine without aura

patients during headache-free periods. They reported that the aerobic exercise increases the beta-

506

507

508

509

510

511

512

513

514 indices involved in migraine headaches were investigated. Therefore, we selected two 515

516

517

518

519

520

521 despite our expectation, the results of the present study showed that 8 weeks of both aerobic

522 training protocols, MOD T and HIGH T, did not affect the serum levels of PACAP in migraine

523 patients. Previous studies have shown that PACAP alterations in migraine patients were two-

524 dimensional when compared with healthy subjects. The quantitative changes in this peptide in the 525

endorphin levels which possibly lead to fewer headache days. PACAP also has been reported to

be antinociceptive in the periphery (Sandor et al., 2009, 2010). One study reported that inhibition

of PACAP expression in the dorsal horn may relieve pain in patients or animals with neuropathic

pain (Mabochi et al., 2004). And, Yamaoka et al. (2017) reported that exercise therapy as a force

treadmill running possibly involves pain relief in spinal nerve ligation (SNL) rats by suppressing

PACAP expression in the spinal cord. They concluded that altered gene expression of PACAP

possibly involved in the mechanism of exercise-induced analgesia for neuropathic pain in rats.

neuropeptides involved in the pathophysiology of migraine, PACAP, and SP, which their role has

recently been reported in migraine headache. According to our review of databases, not any study

has been carried out to investigate the effect of exercise training on serum levels of PACAP in migraine patients. In other words, we first reported the effect of exercise training as aerobic form on serum levels of PACAP in migraine patients. PACAP has been shown to play a role in the pathogenesis of migraine, such as the CGRP (Schytz et al., 2009; Tuka et al., 2013). However, plasma are related to the disease duration. PACAP-38 concentration has been shown significantly 
526 lower in the interictal plasma of the migraineurs as compared with the healthy controls and plasma

527 and significantly higher in the ictal phase relative to the attack-free (Tuka et al., 2013). In this

528 study, significantly lower PACAP-38-LI was determined in the interictal plasma of the

529 migraineurs $(\mathrm{n}=80 ; 24.60 \pm 3.59 \mathrm{fmol} / \mathrm{ml})$ than in that of the healthy volunteers $(\mathrm{n}=40 ; 26.54 \pm 4.43$

$530 \mathrm{fmol} / \mathrm{ml})$. However, the plasma samples from the patients during their migraine attacks $(\mathrm{n}=28)$

531 exhibited a significantly higher PACAP-38 concentration $(27.39 \pm 4.67 \mathrm{fmol} / \mathrm{ml})$ as compared with

532 the interictal samples ( $\mathrm{n}=59 ; 24.91 \pm 3.73 \mathrm{fmol} / \mathrm{ml})$ (Tuka et al., 2013).

533 Therefore, the pattern of PACAP alteration in migraine patients is a sinusoidal and does not have 534 a consistent level in these patients. In the present study, we used 8 weeks aerobic training 535 protocols, which included a number of these attack periods and between attacks periods. 536 Although, patient's migraine state in moment of blood collection may has a critical role in 537 plasma PACAP level, however, in our study, it was not possible to collected blood samples in 538 same migraine phase (ictal or interictal) since time of last training session until blood collection 539 moment must to be same for all subjects. It, therefore, is likely that, in moment of blood 540 collection, some of subjects were in ictal phase and some other were in interictal phase.

541 Therefore, the sinus pattern of the PACAP alteration pattern can be one of the possible reasons

542 for the unchanging it after aerobic training protocols. It was suggested that the effect of aerobic 543 training on serum PACAP would be investigated in separated periods of ictal and interictal.

544 Another possibility for the unchanging PACAP serum levels following aerobic training in 545 migraine patients is neuroprotective and neurotrophic role of PACAP. PACAP is expressed 546 throughout the central nervous system (CNS), such as the hypothalamus, hippocampus, 547 cerebellum, and sensory neurons (Arimura et al., 1994; Hannibal, 2002; Sundler et al., 1996 ), 
548 and this indicates its polyotropic functions in the CNS. PACAP has been shown to act as a nerve

549 hormone, neurotransmitter, and neurotrophic agent (Gonzalez, Basille, Vaudry, Fournier, \&

550 Vaudry, 1997; Lioudyno, Skoglösa, Takei, \& Lindholm, 1998; Vaudry et al., 2000 ). In the adult

551 brain, PACAP prevents apoptotic cell death and can be a regenerative factor in various

552 pathological conditions (Morio, Tatsuno, Hirai, Tamura, \& Saito, 1996). Therefore, considering

553 other roles and functions of PACAPs in the central nervous system, it seems that in migraines,

554 the levels of this protein, on the one hand, are affected by migraine disease, and on the other

555 hand, it requires different expressions for its other functions. Taking this into account, exercise

556 training can affect PACAP roles in the nervous system. In general, it seems that the impact of

557 aerobic training on PACAP in migraine patients will be different from that in healthy people.

558 Therefore, in order to better determine the role of physical activity, such as aerobic training on

559 PACAP in migraines, it is better to study the PACAP level in target tissues and local sites.

560 For the first time, we studied the effect of aerobic training with different intensities (MOD T and

561 HIGH T) on serum SP levels in migraine patients. Interestingly, none of the aerobic training

562 doses, medium, and high intensity, could have any effect on serum SP levels in migraine

563 patients. The role of SP as a vasodilator in the brain has been mentioned in previous studies and

564 was considered as a pain modulator (Beattie, Connor, \& Hagan, 1995 ). In the clinical situations,

565 it has also been shown that, when migraine attacks were triggered, the salivary SP level was

566 increased in migraine patients as compared with the control group (Nicolodi \& Del Bianco,

567 1990). The role of SP in migraine disease is well articulated in previous studies (Fusayasu,

568 Kowa, Takeshima, Nakaso, \& Nakashima, 2007; Jang, Park, Kho, Chung, \& Chung, 2011).

569 Nevertheless, the results of this study showed that aerobic training with moderate and high

570 intensity did not have any significant effect on serum SP levels in migraine patients. In this 
571 regard, it expected that serum SP level would decrease after 8 weeks of aerobic training, but its

572 serum levels did not change. The absence of change in SP after aerobic training may be

573 attributed to other SP roles in the nervous system. Substance P has been shown to have

574 neurotrophic effects and improve memory (Skoff, Zhao, \& Adler, 2009 ). Electrophysiological

575 studies have also shown that SP induces nigral dopaminergic neurons (Skoff et al., 2009). SP

576 also increases dopamine release from dopamine terminals (Gayen, Goswami, \& Mukhopadhyay,

5772011 ), which may be involved in maintaining the integrity of the nerve population (Marolda et

578 al., 2012 ). It also is involved in learning and memory, mood and anxiety, stress mechanisms,

579 and pain (Xu, Xie, Li, Zhang, \& Hou, 2013). Therefore, SP has many different functions in the

580 nervous system, which may require different doses of it. However, the mechanisms underlying

581 SP expression and its influence on migraine pain adaptation to exercise training are not entirely

582 clear. Additional studies should be conducted to elucidate the role of SP in headache in migraine 583 patients.

\section{Conclusion}

586 In summary, the present study showed that aerobic training with moderate and high intensities

587 could significantly reduce migraine pain indices including headache intensity, duration, and

588 frequency of attacks in migraine women. Although, role of PACAP and SP in beneficial effects

589 of aerobic training on migraine indices remain unclear, however, these effects of aerobic training

590 on migraine pain indices can be attributed to various mechanisms that require further studies. In

591 addition, our results showed that the intensity of aerobic training was not the main factor in

592 migraine pain modification, although both aerobic training protocols could be alternative non-

593 pharmacological method to monitor the headache in women with migraine disorder. 


\section{Acknowledgement}

595 The authors would like to thank the Council of Research, University of Allameh Tabataba'i

596 (Iran), for providing financial supports.

597

598 Funding: This work was supported by the Allameh Tabataba'i University (grant numbers:

$599 \mathrm{t} / \mathrm{d} / 62)$.

600

601 Author contributions

602 R. Eslami contributed in the data processing, statistical analysis, and drafted the manuscript; A.

603 Parnow and Z. Pairo conceived of the study, participated in its design and coordination, run

604 training protocols, and gathered data. P. Nikolaidis contributed in data analysis; and B. Knechtle

605 contributed in final edition and managed. All authors have read and approved the final version of

606 the manuscript, and agree with the order of presentation of the authors

607

608 Competing interests

609 The authors declare that there is no conflict of interest.

610

611

612

613

614

615

616 
617

618

619

620

621

622

623

624

625

626

627

628

629

630

631

632

633

634

635

636

637

\section{References}

Amin, A. D., Aristeidou, S., Baraldi, C., Czapinska-Ciepiela, E. K., Ariadni, D. D., Lenola, D. D., Fenech, C., Kampouris, K., Karagiorgis, G., Braschinsky, M. (2018). Mattias LindeThe association between migraine and physical exercise. The Journal of Headache and Pain, 19:83, 1-9. doi: /10.1186/s10194-018-0902-y

Anselmi, B., Baldi, E., Casacci, F., Salmon, S. (1980). Endogenous opioids in cerebrospinal fluid and blood in idiopathic headache sufferers. Headache, 20:294-299.

Arimura, A., Somogyvari-Vigh, A., Weill, C., Fiore, R., Tatsuno, I., Bay, V., \& Brenneman, D. (1994 ). PACAP functions as a neurotrophic factor. Ann N Y Acad Sci, 739(228-43), 228. doi: 10.1111/j.1749-6632.1994.tb19825.x

Beattie, D., Connor, H., \& Hagan, R. (1995 ). Recent developments in tachykinin NK1 receptor antagonists: prospects for the treatment of migraine headache. Can J Physiol Pharmacol, 73(7), 871-877. doi: 10.1139/y95-120

Benbir G, Karadeniz D, Göksan B. The characteristics and subtypes of headache in relation to age and gender in a rural community in Eastern Turkey. Agri. 2012;24:145-152.

Brunton, L. (2006). Goodman \& Gilman's The Pharmacological Basis of Therapeutics, Goodman \& Gilman's The Pharmacological Basis of Therapeutics, pp 547-559

Buse, D. C., Loder, E. W., Gorman, J. A., Stewart, W. F., Reed, M. L., Fanning, K. M., Serrano, D., Lipton, R. B. (2013). Sex Differences in the Prevalence, Symptoms, and Associated Features of Migraine, Probable Migraine and Other Severe Headache: Results of the American Migraine Prevalence and Prevention (AMPP) Study. Headache, 53,1278-1299. 
Buzzi, M., \& Moskowitz, M. (1990). The anti-migraine drug sumatriptan (GR 43175), selectively blocks neurogenic plasma extravasation from blood vessels in dura mater. $B r$. J. Pharmacol, 99, 202-206.

Chang, M., Leeman, S., \& Niall, H. (1971). Amino-acid sequence of substance P. Nat. New Biol, $232,86-87$.

Darabaneanu, S., Overath, C., Rubin, D., Lüthje, S., Sye, W., Niederberger, U., . . Weisser, B. (2011 ). Aerobic exercise as a therapy option for migraine: a pilot study. Int J Sports Med, 32(6), 455-460. doi: doi: 10.1055/s-0030-1269928.

Dittrich, S., Günther, V., Franz, G., Burtscher, M., Holzner, B., \& Kopp, M. (2008 ). Aerobic exercise with relaxation: influence on pain and psychological well-being in female migraine patients. Clin J Sport Med, 18(4), 363-365. doi: 10.1097/JSM.0b013e31817efac9

Ferrari, M. (1998). Migraine. Lancet, 351, 1043-1051.

Fusayasu, E., Kowa, H., Takeshima, T., Nakaso, K., \& Nakashima, K. (2007). Increased plasma substance P and CGRP levels, and high ACE activity in migraineurs during headachefree periods. Pain, 128, 209-214. doi: 10.1016/j.pain.2006.09.017

Gantenbein, A., Afra, J., Jenni, W., \& Sándor, P. (2012). Complementary and alternative treatments for migraine. Techniques in Regional Anesthesia and Pain Management, 16(1), 76-81.

Gayen, A., Goswami, S., \& Mukhopadhyay, C. (2011 ). NMR evidence of GM1-induced conformational change of Substance P using isotropic bicelles. Biochim Biophys Acta, 1808(1), 127-139. doi: 10.1016/j.bbamem.2010.09.023 
660

661

662

663

664

665

666

667

668

669

670

671

672

673

674

675

676

677

678

679

680

681

682

Goadsby, P., Lipton, R., \& Ferrari, M. (2002). Migraine: current understanding and treatment. $N$ Engl J Med, 346, 257-270.

Gonzalez, B., Basille, M., Vaudry, D., Fournier, A., \& Vaudry, H. (1997 ). Pituitary adenylate cyclase-activating polypeptide promotes cell survival and neurite outgrowth in rat cerebellar neuroblasts. Neuroscience, 78(2), 419-430.

Grimm, L., Douglas, D., \& Hanson, P. (1981). Aerobic training in the prophylaxis of migraine. Med Sci Sports Exer, 15.

Guillemin, R., Vargo, T., Rossier, J., Minick, S., Ling, N., Rivier, C., Vale, W., Bloom, F. (1977). Beta-endorphin and adrenocorticotropin are selected concomitantly by the pituitary gland. Science 197:1367-1369.

Hannibal, J. (2002). Pituitary adenylate cyclase-activating peptide in the rat central nervous system: an immunohistochemical and in situ hybridization study. J. Comp. Neurol, 453, $389-417$.

Henry P, Auray JP, Gaudin AF, et al. Prevalence and clinical characteristics of migraine in France. Neurology. 1999;53:537-542.

Hindiyeh, N., Krusz, J., \& Cowan, R. (2013 ). Does exercise make migraines worse and tension type headaches better? Curr Pain Headache Rep, 17(12). doi: 10.1007/s11916-013-03805

Irby, M. B., Bond, D. S., Lipton, R. B., Nicklas, B., Houle, T. T., and Penzien, D. B. (2016) Aerobic Exercise for Reducing Migraine Burden: Mechanisms, Markers, and Models of Change Processes. Headache., 56(2): 357-369. doi:10.1111/head.12738.

Jang, M., Park, J., Kho, H., Chung, S., \& Chung, J. (2011). Plasma and saliva levels of nerve growth factor and neuropeptides in chronic migraine patients. Oral Dis, 17, 187-193. 
Kaminsky, L. (2014). ACSM's health-related physical fitness assessment manual (K. LA Ed.): Lippincott Williams \& Wilkins.

Kim BK, Chung YK, Kim JM, Lee KS, Chu MK. (2013). Prevalence, clinical characteristics and disability of migraine and probable migraine: A nationwide population-based survey in Korea. Cephalalgia.;Epub ahead of print.

Köseoglu E, Akboyraz A, Soyuer A, Ersoy AÖ (2003). Aerobic exercise and plasma beta endorphin levels in patients with migrainous headache without aura. Cephalalgia 23:972976.

Krøll, L.S., Hammarlund, C.S., Linde, M., et al., (2018). The effects of aerobic exercise for persons with migraine and co-existing tension-type headache and neck pain. A randomized, controlled, clinical trial. Cephalalgia, :333102417752119. [Epub ahead of print].

Lioudyno, M., Skoglösa, Y., Takei, N., \& Lindholm, D. (1998). Pituitary adenylate cyclaseactivating polypeptide (PACAP) protects dorsal root ganglion neurons from death and induces calcitonin gene-related peptide (CGRP) immunoreactivity in vitro. J. Neurosci. Res, 51, 243-256. doi: 10.1002/(SICI)1097-4547(19980115)51:2<243::AIDJNR13>3.0.CO;2-9

Lockett, D., \& Campbell, J. (1992 ). The effects of aerobic exercise on migraine. Headache. Jan;():50-4., 32(1), 50-54.

Maeda, S., Miyauchi, T., Kakiyama, T., Sugawara, J., Iemitsu, M., Irukayama-Tomobe, Y., . . . Matsuda, M. (2001 ). Effects of exercise training of 8 weeks and detraining on plasma levels of endothelium-derived factors, endothelin-1 and nitric oxide, in healthy young humans. Life Sci, 69(9), 1005-1016. doi: 10.1016/s0024-3205(01)01192-4 
Marolda, R., Ciotti, M., Matrone, C., Possenti, R., Calissano, P., Cavallaro, S., \& Severini, C. (2012 ). Substance P activates ADAM9 mRNA expression and induces $\alpha$-secretasemediated amyloid precursor protein cleavage. Neuropharmacology, 62(5-6), 1954-1963. doi: 10.1016/j.neuropharm.2011.12.025

Morio, H., Tatsuno, I., Hirai, A., Tamura, Y., \& Saito, Y. (1996). Pituitary adenylate cyclaseactivating polypeptide protects rat-cultured cortical neurons from glutamate-induced cytotoxicity. Brain Res. Brain Res, 741, 82-88. doi: 10.1016/s0006-8993(96)00920-1

Moskowitz, M. (1993). Neurogenic inflammation in the pathophysiology and treatment of migraine., ,: S16-S20. Neurology, 43(6 Suppl 3), S16-20.

Narin, S., Pinar, L., Erbas, D., Oztürk, V., \& Idiman, F. (2003 ). The effects of exercise and exercise-related changes in blood nitric oxide level on migraine headache. Clin Rehabil. Sep;():: 17(6), 624-630. doi: 10.1191/0269215503cr657oa

Nicolodi, M., \& Del Bianco, E. (1990). Sensory neuropeptides (substance P, calcitonin generelated peptide) and vasoactive intestinal polypeptide in human saliva: their pattern in migraine and cluster headache. Cephalalgia, 10, 39-50.

norlander, E., Cider, A., Carlsson, J., \& Linde, M. (2007). Improvement of exercise capacity in patients with migraine-methodological considerations. Cephalalgia, 27, 575-579.

Onuoha, G., Nicholls, D., Patterson, A., \& Beringer, T. (1998). neuropeptide secretion in exercise. Neuropeptides, 32(4), 319-325.

Scheef, L., Jankowski, J., Daamen, M., Weyer, G., Klingenberg, M., Renner, J., . . Boecker, H. (2012). An fMRI study on the acute effects of exercise on pain processing in trained athletes. Pain, 153(8), 1702-1714. 
Schytz, H., Birk, S., Wienecke, T., Kruuse, C., Olesen, J., \& Ashina, M. ( 2009). PACAP38 induces migraine-like attacks in patients with migraine without aura. Brain, 132, 16-25.

Skoff, A., Zhao, C., \& Adler, J. (2009 ). Interleukin-1alpha regulates substance P expression and release in adult sensory neurons. Exp Neurol, 217(2), 395-400. doi: 10.1016/j.expneurol.2009.03.022

Sandor, K., Kormos, V., Botz, B., Imreh, A., Bolcskei, K., Gaszner, B., Markovics, A., Szolcsanyi, J., Shintani, N., Hashimoto, H., Baba, A., Reglodi, D., Helyes, Z. (2010). Impaired nocifensive behaviours and mechanical hyperalgesia, but enhanced thermal allodynia in pituitary adenylate cyclase-activating polypeptide deficient mice. Neuropeptides 44, 363-371.

Sandor, K., Bolcskei, K., McDougall, J.J., Schuelert, N., Reglodi, D., Elekes, K., Petho, G., Pinter, E., Szolcsanyi, J., Helyes, Z. (2009). Divergent peripheral effects of pituitary adenylate cyclase-activating polypeptide-38 on nociception in rats and mice. Pain 141, $143-150$.

Steiner TJ, Scher AI, Stewart WF, Kolodner K, Liberman J, Lipton RB. The prevalence and disability burden of adult migraine in England and their relationships to age, gender ethnicity. Cephalalgia. 2003;23:519-527.

Sundler, F., Ekblad, E., Hannibal, J., Moller, K., Zhang, Y., Mulder, H., . . . Uddman, R. (1996 ). Pituitary adenylate cyclase-activating peptide in sensory and autonomic ganglia: localization and regulation. Ann N Y Acad Sci, 805(410-28), 410. doi: 10.1111/j.17496632.1996.tb17501.x

Tajti, J., Szok, D., Majláth, Z., Tuka, B., \& Csáti, A. (2015). Migraine and neuropeptides. Neuropeptides, 52 19-30. 
751 Tuka, B., Helyes, Z., Markovics, A., Bagoly, T., Szolcsanyi, J., Szabo, N., . . Tajti, J. (2013).

752

753

754

755

756

757

758

759

760

761

762

763

764

765

766

767

768

769

770

771

772

773

Alterations in PACAP-38-like immunoreactivity in the plasma during ictal and interictal periods of migraine patients. Cephalalgia, 33, 1085-1095.

Varkey E, C. A., Carlsson J, Linde M. (2009 ). A study to evaluate the feasibility of an aerobic exercise program in patients with migraine. Headache, 49(4), 563-570. doi: doi: 10.1111/j.1526-4610.2008.01231.x

Varkey, E., Cider, A., Carlsson, J., \& Linde, M. (2011 ). Exercise as migraine prophylaxis: a randomized study using relaxation and topiramate as controls. Cephalalgia, 31(14), 1428-1438. doi: doi: 10.1177/0333102411419681

Vaudry, D., Gonzalez, B., Basille, M., Yon, L., Fournier, A., \& Vaudry, H. (2000 ). Pituitary adenylate cyclase-activating polypeptide and its receptors: from structure to functions. Pharmacol Rev, 52(2), 269-324.

Xu, D., Xie, X., Li, S., Zhang, J., \& Hou, L. (2013). Function of Substance P and Substance P Receptor in Exercise. International Journal of Applied Physics and Mathematics, 3(1), $78-81$

Yamaoka S., Oshima Y., Horiuchi H., Morino T., Hino M., Miura H., and Ogata t., (2017). Altered Gene Expression of RNF34 and PACAP Possibly Involved in Mechanism of Exercise-Induced Analgesia for Neuropathic Pain in Rats. Int. J. Mol. Sci. 2017, 18, 1962; doi:10.3390/ijms18091962 
774 Table 1: Demographic characteristics of subjects with different groups in initial state.

\begin{tabular}{|c|c|c|c|}
\hline & $\begin{array}{l}\mathrm{CON} \\
(n=15) \\
(\text { mean } \pm \text { SD })\end{array}$ & $\begin{array}{l}\text { MOD T } \\
(n=15) \\
(\operatorname{mean} \pm \text { SD })\end{array}$ & $\begin{array}{l}\text { HIGH T } \\
(n=15) \\
(\text { mean } \pm \text { SD })\end{array}$ \\
\hline Age (years) & $32.44 \pm 5.74$ & $38.41 \pm 6.20$ & $25.16 \pm 6.08$ \\
\hline Weight (kg) & $61.98 \pm 10.55$ & $69.75 \pm 14.42$ & $57.12 \pm 10.84$ \\
\hline BMI $\left(\mathrm{kg} / \mathrm{m}^{2}\right)$ & $24.10 \pm 4.42$ & $26.93 \pm 5.65$ & $21.00 \pm 3.1$ \\
\hline $\mathrm{VO}_{2} \mathrm{max}(\mathrm{ml} / \mathrm{kg} / \mathrm{min})$ & $36.89 \pm 11.23$ & $28.69 \pm 4.48$ & $33.90 \pm 8.83$ \\
\hline
\end{tabular}

$\begin{array}{llll}\text { Body Fat (\%) } & 28.54 \pm 6.89 & 29.87 \pm 10.19 & 21.60 \pm 6.57\end{array}$


799

800

801

802

803

804

805

806

807

808

809

810

811

812

813

814

815

816

817

818

819

820

821

822

823

824

825

826

827

828

829

830

831

832

833

834

835

Table 2: Aerobic training protocol with different intensities

\begin{tabular}{|c|c|c|c|c|c|c|c|c|c|}
\hline \multirow[t]{2}{*}{ Groups } & \multirow{2}{*}{$\begin{array}{l}\text { Training } \\
\text { Variables }\end{array}$} & \multicolumn{8}{|c|}{ Weeks } \\
\hline & & One & Two & tree & four & five & six & seven & eight \\
\hline \multirow{5}{*}{ MOD T } & $\begin{array}{l}\text { Intensity } \\
(\mathrm{RPE})\end{array}$ & 8 & 12 & 12 & 13 & 14 & 14 & 15 & 15 \\
\hline & $\begin{array}{l}\text { Intensity } \\
(\% \text { HRmax })\end{array}$ & 60 & 62 & 72 & 78 & 80 & 80 & 82 & 82 \\
\hline & $\begin{array}{l}\text { Intensity } \\
\left(\% \mathrm{VO}_{2 \mathrm{max}}\right)\end{array}$ & 45 & 46 & 50 & 60 & 68 & 68 & 70 & 70 \\
\hline & Time $(\min )$ & 7 & 18 & 25 & 30 & 35 & 40 & 40 & 40 \\
\hline & $\begin{array}{l}\text { Work load } \\
\text { (RPE*time) }\end{array}$ & 56 & 216 & 300 & 390 & 490 & 560 & 600 & 600 \\
\hline \multirow{5}{*}{ HIGH T } & Intensity & 10 & 12 & 12 & 14 & 16 & 16 & 17 & 17 \\
\hline & $\begin{array}{l}\text { Intensity } \\
(\% H R m a x)\end{array}$ & 65 & 79 & 82 & 88 & 92 & 92 & 92 & 95 \\
\hline & $\begin{array}{l}\text { Intensity } \\
\left(\% \mathrm{VO}_{2} \text { max }\right)\end{array}$ & 55 & 70 & 72 & 80 & 83 & 88 & 88 & 90 \\
\hline & Time (min) & 5 & 10 & 15 & 20 & 25 & 30 & 30 & 30 \\
\hline & $\begin{array}{l}\text { Work load } \\
\text { (RPE*time) }\end{array}$ & 50 & 120 & 180 & 280 & 400 & 480 & 510 & 510 \\
\hline
\end{tabular}


836 Table 3: The comparison of variables in pre and post time and between three groups (time effects 837 and time* group interaction effects).

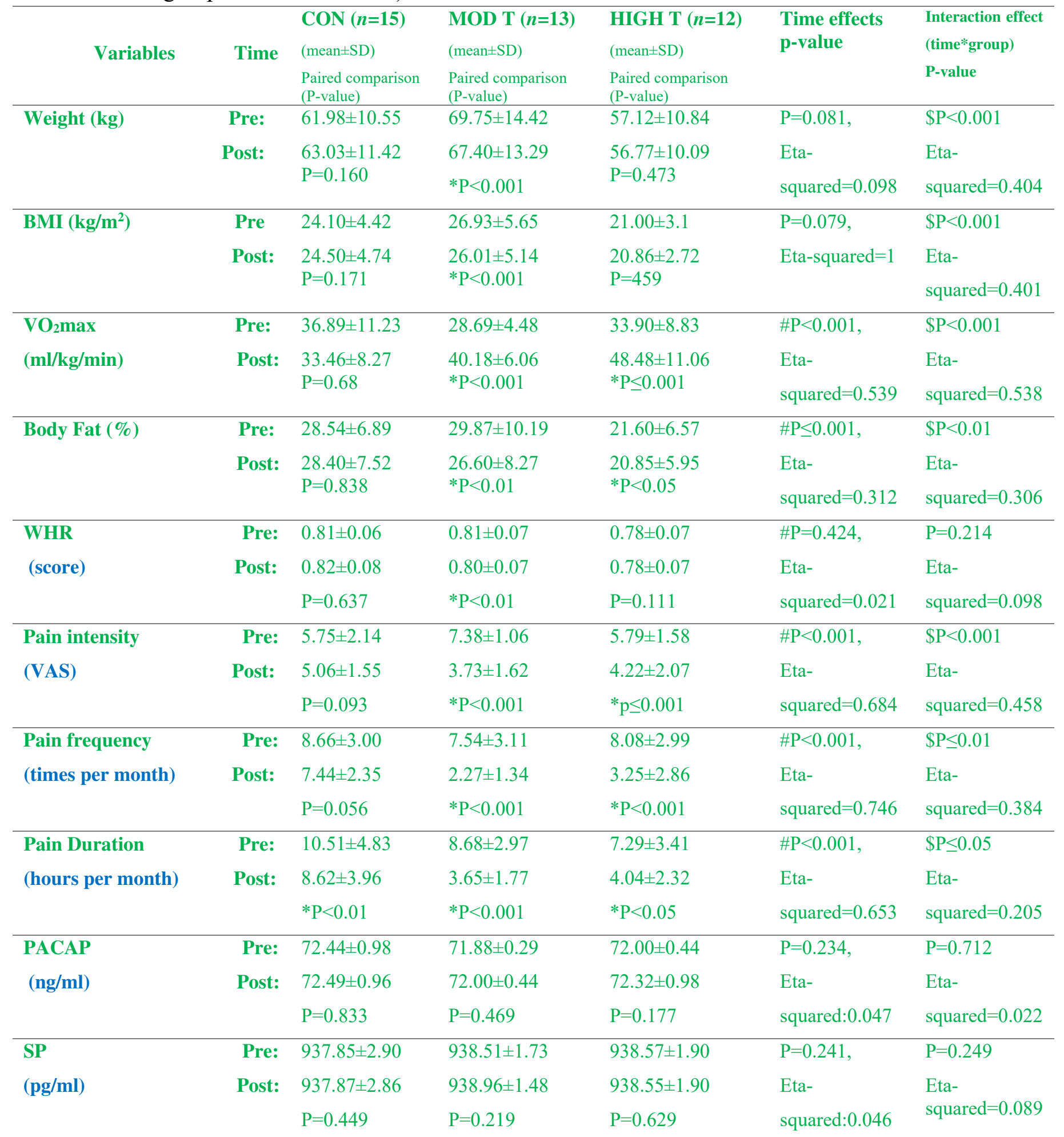

838 CON: Control; MOD T; Moderate-intensity aerobic training; HIGH T: High-intensity aerobic training

$839 *$ Significant pre-post different

$840 \quad$ \# Significant time effect 


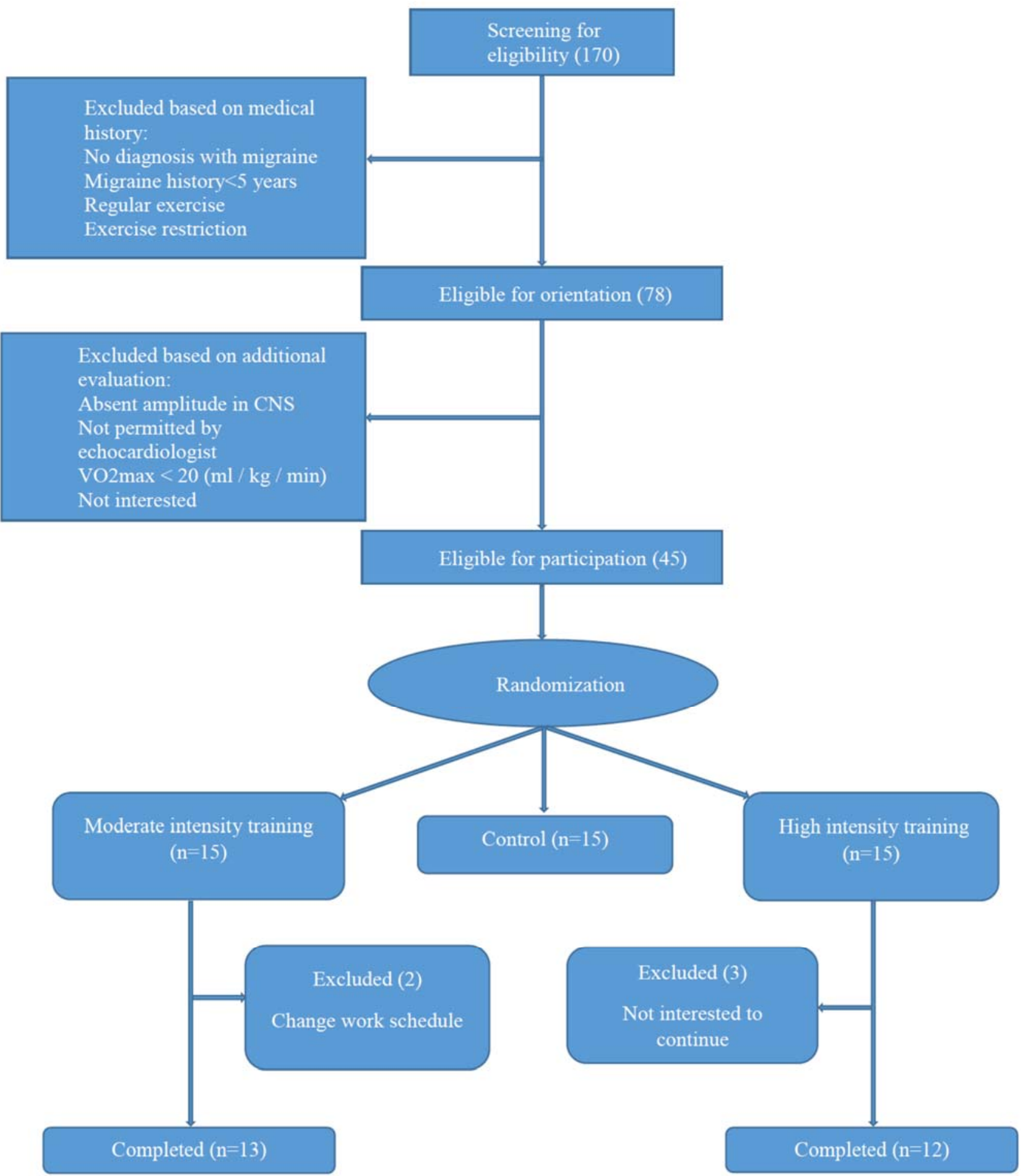

842 Figure 1: Participant recruitment flow chart.

843

844 

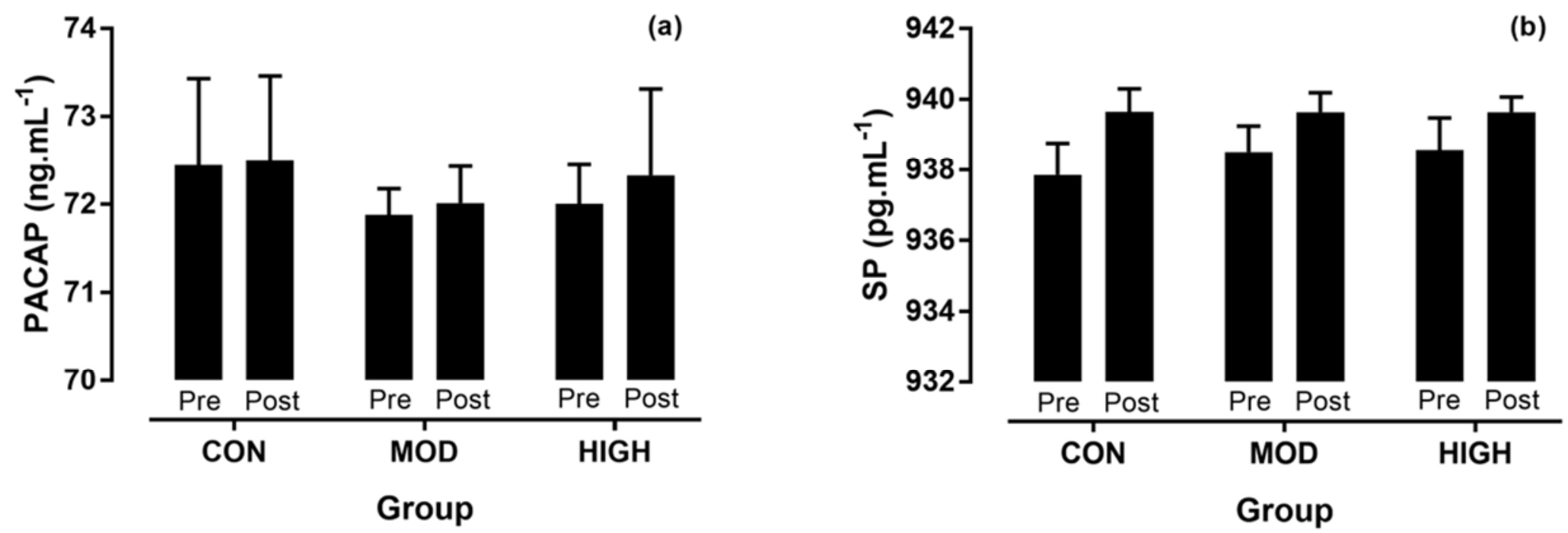

Figure2: PACAP (a) and SP (b) contents in three groups in pre and post training. CON: Control group; MOD: Moderate intense aerobic

847 training group; HIGH: High intense aerobic training. 